\title{
Oral Medicine Specialist
}

National Cancer Institute

\section{Source}

National Cancer Institute. Oral Medicine Specialist. NCI Thesaurus. Code C63742.

A dentist specializing in the diagnosis and non-surgical management of medically-related disorders or conditions affecting the oral and maxillofacial region. 